\title{
Positive solutions for a class of fractional differential equations at resonance
}

\author{
Yanqiang $\mathrm{Wu}^{*}$ and Wenbin Liu
}

${ }^{\text {*Correspondence: }}$ wyq1976819@126.com

College of Sciences, China University of Mining and

Technology, Xuzhou, 221008, China

\begin{abstract}
In this paper, by using the Leggett-Williams norm-type theorem, we consider a $m$-point boundary value problem for a class of fractional differential equations at resonance. A new result on the existence of solutions for above problem is obtained.

MSC: $34 \mathrm{~A} 08 ; 34 \mathrm{~B} 15$

Keywords: fractional differential equation; $m$-point boundary value problem; Leggett-Williams norm-type theorem; resonance
\end{abstract}

\section{Introduction}

The subject of fractional calculus has gained significant interest and been a valuable tool for both science and engineering (see [1-3]). In recent years, the fractional boundary value problems (FBVPs for short) have been considered by many authors (see [4-10] and the references therein). For example, Bai studied a FBVP at non-resonance with $1<\alpha \leq 2$ (see [10]). FBVPs at resonance were studied by Kosmatov (see [11]) and Jiang (see [12]). But the positive solutions for FBVPs at resonance were studied very few. In [13], Yang and Wang considered the positive solutions of the following FBVP:

$$
\left\{\begin{array}{l}
-D_{0^{+}}^{\alpha} x(t)=f(t, x(t)), \quad t \in[0,1] \\
x(0)=0, \quad x^{\prime}(0)=x^{\prime}(1)
\end{array}\right.
$$

In [14], Chen and Tang studied the positive solution of FBVP as follows:

$$
\left\{\begin{array}{l}
D_{0^{+}}^{\alpha} x(t)=f(t, x(t)), \quad t \in[0,+\infty), \\
x(0)=x^{\prime}(0)=x^{\prime \prime}(0)=0, \quad D_{0^{+}}^{\alpha-1} x(0)=\lim _{t \rightarrow+\infty} D_{0^{+}}^{\alpha-1} x(t) .
\end{array}\right.
$$

However, to the best of our knowledge, the fractional differential equations with $m$-point boundary conditions at resonance have not been considered. Motivated by the papers above, we consider the existence of positive solutions for a $m$-point FBVP of the form

$$
\left\{\begin{array}{l}
-D_{0^{+}}^{\alpha} x(t)=f(t, x(t)), \quad t \in[0,1], \\
x^{\prime}(0)=0, \quad x(1)=\sum_{i=1}^{m-2} \beta_{i} x\left(\eta_{i}\right),
\end{array}\right.
$$

where $D_{0^{+}}^{\alpha}$ denotes the standard Caputo fractional differential operator of order $\alpha$, $1<\alpha \leq 2, \beta_{i} \in \mathbb{R}^{+}, \sum_{i=1}^{m-2} \beta_{i}=1,0<\eta_{1}<\eta_{2}<\cdots<\eta_{m-2}<1$, and $f:[0,1] \times \mathbb{R} \rightarrow \mathbb{R}$ is continuous. Obviously, FBVP (1.1) happens to be at resonance under the condition $\sum_{i=1}^{m-2} \beta_{i}=1$.

(c) 2015 Wu and Liu. This article is distributed under the terms of the Creative Commons Attribution 4.0 International License (http://creativecommons.org/licenses/by/4.0/), which permits unrestricted use, distribution, and reproduction in any medium, provided you give appropriate credit to the original author(s) and the source, provide a link to the Creative Commons license, and indicate if changes were made. 
The rest of this paper is organized as follows. Section 2 contains some necessary notations, definitions and lemmas. In Section 3, we establish a theorem on the existence of positive solutions for FBVP (1.1) under some restrictions of $f$, basing on the coincidence degree theory due to [15]. Finally, in Section 4, an example is given to illustrate the main result.

\section{Preliminaries}

For convenience of the reader, we present some definitions, notations, and preliminary statements, which can be found in $[2,16,17]$.

Let $X$ and $Y$ be real Banach spaces, $L: \operatorname{dom} L \subset X \rightarrow Y$ be a Fredholm operator with index zero, where the index of a Fredholm operator $L$ is defined by

$$
\text { Index } L:=\operatorname{dim} \operatorname{Ker} L-\operatorname{dim} \operatorname{Coker} L \text {. }
$$

Suppose $P: X \rightarrow X, Q: Y \rightarrow Y$ be continuous linear projectors such that

$$
\begin{aligned}
& \operatorname{Im} P=\operatorname{Ker} L, \quad \operatorname{Ker} Q=\operatorname{Im} L, \\
& X=\operatorname{Ker} L \oplus \operatorname{Ker} P, \quad Y=\operatorname{Im} L \oplus \operatorname{Im} Q .
\end{aligned}
$$

Thus, we see that

$$
\left.L\right|_{\operatorname{dom} L \cap \operatorname{Ker} P}: \operatorname{dom} L \cap \operatorname{Ker} P \rightarrow \operatorname{Im} L
$$

is invertible. We denote the inverse by $K_{P}$. Moreover, by virtue of $\operatorname{dim} \operatorname{Im} Q=\operatorname{codim} \operatorname{Im} L$, there exists an isomorphism $J: \operatorname{Im} Q \rightarrow \operatorname{Ker} L$. Then we know that the operator equation $L x=N x$ is equivalent to

$$
x=(P+J Q N) x+K_{P}(I-Q) N x,
$$

where $N: X \rightarrow Y$ be a nonlinear operator.

If $\Omega$ is an open bounded subset of $X$ such that $\operatorname{dom} L \cap \bar{\Omega} \neq \emptyset$, then the map $N: X \rightarrow Y$ will be called $L$-compact on $\bar{\Omega}$ if $Q N: \bar{\Omega} \rightarrow Y$ is bounded and $K_{P}(I-Q) N: \bar{\Omega} \rightarrow X$ is compact.

Let $C$ be a cone in $X$. Then $C$ induces a partial order in $X$ by

$$
x \leq y \text { if and only if } y-x \in C .
$$

Lemma 2.1 (see [15]) Let $C$ be a cone in $X$. Then for every $u \in C \backslash\{0\}$ there exists a positive number $\sigma(u)$ such that

$$
\|x+u\| \geq \sigma(u)\|x\|
$$

for all $x \in C$.

Let $\gamma: X \rightarrow C$ be a retraction, that is, a continuous mapping such that $\gamma(x)=x$ for all $x \in C$. Set

$$
\Psi:=P+J Q N+K_{P}(I-Q) N
$$


and

$$
\Psi_{\gamma}:=\Psi \circ \gamma
$$

Lemma 2.2 (see [15]) Let $C$ be a cone in $X$ and $\Omega_{1}, \Omega_{2}$ be open bounded subsets of $X$ with $\bar{\Omega}_{1} \subset \Omega_{2}$ and $C \cap\left(\bar{\Omega}_{2} \backslash \Omega_{1}\right) \neq \emptyset$. Assume that the following conditions are satisfied:

(1) $L: \operatorname{dom} L \subset X \rightarrow Y$ be a Fredholm operator of index zero and $N: X \rightarrow Y$ be $L$-compact on every bounded subset of $X$,

(2) $L x \neq \lambda N x$ for every $(x, \lambda) \in\left[C \cap \partial \Omega_{2} \cap \operatorname{dom} L\right] \times(0,1)$,

(3) $\gamma$ maps subsets of $\bar{\Omega}_{2}$ into bounded subsets of $C$,

(4) $\operatorname{deg}\left(\left.[I-(P+J Q N) \gamma]\right|_{\operatorname{Ker} L}, \operatorname{Ker} L \cap \Omega_{2}, 0\right) \neq 0$,

(5) there exists $u_{0} \in C \backslash\{0\}$ such that $\|x\| \leq \sigma\left(u_{0}\right)\|\Psi x\|$ for $x \in C\left(u_{0}\right) \cap \partial \Omega_{1}$, where $C\left(u_{0}\right)=\left\{x \in C: \mu u_{0} \leq x\right.$ for some $\left.\mu>0\right\}$ and $\sigma\left(u_{0}\right)$ is such that $\left\|x+u_{0}\right\| \geq \sigma\left(u_{0}\right)\|x\|$ for every $x \in C$,

(6) $(P+J Q N) \gamma\left(\partial \Omega_{2}\right) \subset C$,

(7) $\Psi_{\gamma}\left(\bar{\Omega}_{2} \backslash \Omega_{1}\right) \subset C$.

Then the equation $L x=N x$ has at least one solution in $C \cap\left(\bar{\Omega}_{2} \backslash \Omega_{1}\right)$.

Definition 2.3 (see [17]) The Riemann-Liouville fractional integral operator of order $\alpha>$ 0 of a function $x$ is given by

$$
I_{0^{+}}^{\alpha} x(t)=\frac{1}{\Gamma(\alpha)} \int_{0}^{t}(t-s)^{\alpha-1} x(s) d s,
$$

provided that the right side integral is pointwise defined on $(0,+\infty)$.

Definition 2.4 (see [17]) The Caputo fractional derivative of order $\alpha>0$ of a continuous function $x$ is given by

$$
D_{0^{+}}^{\alpha} x(t)=\frac{1}{\Gamma(n-\alpha)} \int_{0}^{t}(t-s)^{n-\alpha-1} x^{(n)}(s) d s,
$$

where $n$ is the smallest integer greater than or equal to $\alpha$, provided that the right side integral is pointwise defined on $(0,+\infty)$.

Lemma 2.5 (see [18]) For $\alpha>0$, the general solution of the Caputo fractional differential equation

$$
D_{0^{+}}^{\alpha} x(t)=0
$$

is

$$
x(t)=c_{0}+c_{1} t+c_{2} t^{2}+\cdots+c_{n-1} t^{n-1},
$$

where $c_{i} \in \mathbb{R}, i=0,1, \ldots, n-1$, here $n$ is the smallest integer greater than or equal to $\alpha$.

Lemma 2.6 (see [18]) Suppose that $D_{0^{+}}^{\alpha} x \in C[0,1], \alpha>0$. Then

$$
I_{0^{+}}^{\alpha} D_{0^{+}}^{\alpha} x(t)=x(t)+c_{0}+c_{1} t+c_{2} t^{2}+\cdots+c_{n-1} t^{n-1},
$$

where $c_{i} \in \mathbb{R}, i=0,1, \ldots, n-1$, here $n$ is the smallest integer greater than or equal to $\alpha$. 
In this paper, we take $X=Y=C[0,1]$ with the norm $\|x\|_{\infty}=\max _{t \in[0,1]}|x(t)|$.

Define the operator $L: \operatorname{dom} L \subset X \rightarrow Y$ by

$$
L x=-D_{0^{+}}^{\alpha} x
$$

where

$$
\operatorname{dom} L=\left\{x \in X: D_{0^{+}}^{\alpha} x \in Y, x^{\prime}(0)=0, x(1)=\sum_{i=1}^{m-2} \beta_{i} x\left(\eta_{i}\right)\right\} .
$$

Let $N: X \rightarrow Y$ be the Nemytskii operator

$$
N x(t)=f(t, x(t)), \quad \forall t \in[0,1] .
$$

Then FBVP (1.1) is equivalent to the operator equation

$$
L x=N x, \quad x \in \operatorname{dom} L .
$$

\section{Main result}

In this section, a theorem on the existence of positive solutions for FBVP (1.1) will be given.

For simplicity of notation, we set

$$
l_{i}(s)= \begin{cases}(1-s)^{\alpha-1}-\left(\eta_{i}-s\right)^{\alpha-1}, & 0 \leq s \leq \eta_{i} \leq 1, \\ (1-s)^{\alpha-1}, & 0 \leq \eta_{i} \leq s \leq 1,\end{cases}
$$

and

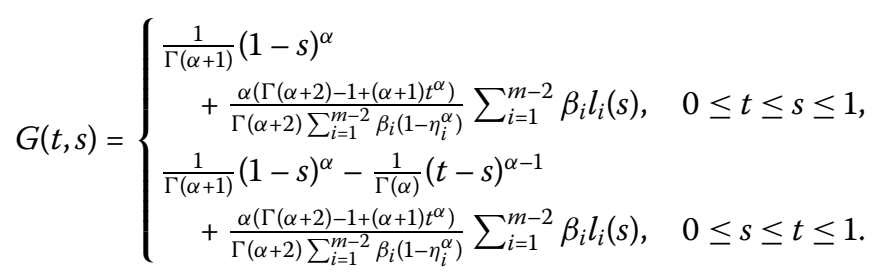

Obviously, $\max _{0 \leq s \leq 1} \sum_{i=1}^{m-2} \beta_{i} l_{i}(s) \leq 1$. We denote

$$
\kappa:=\min \left\{1, \frac{\sum_{i=1}^{m-2} \beta_{i}\left(1-\eta_{i}^{\alpha}\right)}{\alpha}, \frac{1}{\max _{t, s \in[0,1]} G(t, s)}\right\} .
$$

Thus, one has

$$
1-\frac{\kappa \alpha \sum_{i=1}^{m-2} \beta_{i} l_{i}(s)}{\sum_{i=1}^{m-2} \beta_{i}\left(1-\eta_{i}^{\alpha}\right)} \geq 0, \quad 1-\kappa G(t, s) \geq 0 .
$$

Theorem 3.1 Let $f:[0,1] \times \mathbb{R} \rightarrow \mathbb{R}$ be continuous. Suppose that:

$\left(\mathrm{H}_{1}\right)$ there exist nonnegative functions $a, b \in X$ with $\frac{\Gamma(\alpha+1)}{2} b_{1}<1$ such that

$$
|f(t, u)| \leq a(t)+b(t)|u|, \quad \forall t \in[0,1], u \in \mathbb{R}
$$

where $b_{1}=\|b\|_{\infty}$, 
$\left(\mathrm{H}_{2}\right)$ there exists a constant $B>0$ such that

$$
u f(t, u)<0, \quad \forall t \in[0,1],|u|>B
$$

$\left(\mathrm{H}_{3}\right) f(t, u)>-\kappa u$, for all $(t, u) \in[0,1] \times[0, \infty)$,

$\left(\mathrm{H}_{4}\right)$ there exist $r \in(0,+\infty), t_{0} \in[0,1], M \in(0,1)$ and continuous function $h:(0, r] \rightarrow$ $[0, \infty)$ such that $f(t, u) \geq h(u)$ for all $t \in[0,1], u \in(0, r]$, and $\frac{h(u)}{u}$ is non-increasing on $(0, r]$ with

$$
\frac{h(r)}{r} \int_{0}^{1} G\left(t_{0}, s\right) d s \geq \frac{1-M}{M} .
$$

Then FBVP (1.1) has at least one solution in X.

Now, we begin with some lemmas that are useful in what follows.

Lemma 3.2 Let L be defined by (2.1), then

$$
\begin{aligned}
& \operatorname{Ker} L=\{x \in X: x(t)=c, \forall t \in[0,1], c \in \mathbb{R}\}, \\
& \operatorname{Im} L=\left\{y \in Y: \sum_{i=1}^{m-2} \beta_{i} \int_{0}^{1} l_{i}(s) y(s) d s=0\right\} .
\end{aligned}
$$

Proof By Lemma $2.5, D_{0^{+}}^{\alpha} x(t)=0$ has solution

$$
x(t)=c_{0}+c_{1} t, \quad c_{0}, c_{1} \in \mathbb{R} .
$$

Combining with the boundary conditions of FBVP (1.1), one sees that (3.2) holds.

For $y \in \operatorname{Im} L$, there exists $x \in \operatorname{dom} L$ such that $y=L x \in Y$. By Lemma 2.6, we have

$$
x(t)=-\frac{1}{\Gamma(\alpha)} \int_{0}^{t}(t-s)^{\alpha-1} y(s) d s+c_{0}+c_{1} t, \quad c_{0}, c_{1} \in \mathbb{R} .
$$

Then we get

$$
x^{\prime}(t)=-\frac{1}{\Gamma(\alpha-1)} \int_{0}^{t}(t-s)^{\alpha-2} y(s) d s+c_{1} .
$$

By the boundary conditions of FBVP (1.1), we see that $y$ satisfies

$$
\int_{0}^{1}(1-s)^{\alpha-1} y(s) d s=\sum_{i=1}^{m-2} \beta_{i} \int_{0}^{\eta_{i}}\left(\eta_{i}-s\right)^{\alpha-1} y(s) d s .
$$

That is,

$$
\sum_{i=1}^{m-2} \beta_{i} \int_{0}^{1} l_{i}(s) y(s) d s=0 .
$$

On the other hand, suppose $y \in Y$ and satisfies (3.4). Let $x(t)=-I_{0^{+}}^{\alpha} y(t)+x(0)$, then $x \in \operatorname{dom} L$ and $D_{0^{+}}^{\alpha} x(t)=-y(t)$. Thus, $y \in \operatorname{Im} L$. Hence (3.3) holds. The proof is complete. 
Lemma 3.3 Let $L$ be defined by (2.1), then $L$ is a Fredholm operator of index zero, and the linear continuous projector operators $P: X \rightarrow X$ and $Q: Y \rightarrow Y$ can be defined as

$$
\begin{aligned}
& P x(t)=\int_{0}^{1} x(s) d s, \quad \forall t \in[0,1] \\
& Q y(t)=\frac{\alpha}{\sum_{i=1}^{m-2} \beta_{i}\left(1-\eta_{i}^{\alpha}\right)} \sum_{i=1}^{m-2} \beta_{i} \int_{0}^{1} l_{i}(s) y(s) d s, \quad \forall t \in[0,1] .
\end{aligned}
$$

Furthermore, the operator $K_{P}: \operatorname{Im} L \rightarrow \operatorname{dom} L \cap \operatorname{Ker} P$ can be written by

$$
K_{P} y(t)=\int_{0}^{1} k(t, s) y(s) d s, \quad \forall t \in[0,1]
$$

where

$$
k(t, s)= \begin{cases}\frac{1}{\Gamma(\alpha+1)}(1-s)^{\alpha}, & 0 \leq t \leq s \leq 1 \\ \frac{1}{\Gamma(\alpha+1)}(1-s)^{\alpha}-\frac{1}{\Gamma(\alpha)}(t-s)^{\alpha-1}, & 0 \leq s \leq t \leq 1\end{cases}
$$

Proof Obviously, $\operatorname{Im} P=\operatorname{Ker} L$ and $P^{2} x=P x$. It follows from $x=(x-P x)+P x$ that $X=$ $\operatorname{Ker} P+\operatorname{Ker} L$. By a simple calculation, one obtain $\operatorname{Ker} L \cap \operatorname{Ker} P=\{0\}$. Thus, we get

$$
X=\operatorname{Ker} L \oplus \operatorname{Ker} P .
$$

For $y \in Y$, we have

$$
Q^{2} y=Q(Q y)=Q y \cdot \frac{\alpha}{\sum_{i=1}^{m-2} \beta_{i}\left(1-\eta_{i}^{\alpha}\right)} \sum_{i=1}^{m-2} \beta_{i} \int_{0}^{1} l_{i}(s) d s=Q y .
$$

Let $y=(y-Q y)+Q y$, where $y-Q y \in \operatorname{Ker} Q, Q y \in \operatorname{Im} Q$. It follows from $\operatorname{Ker} Q=\operatorname{Im} L$ and $Q^{2} y=Q y$ that $\operatorname{Im} Q \cap \operatorname{Im} L=\{0\}$. Then one has

$$
Y=\operatorname{Im} L \oplus \operatorname{Im} Q .
$$

Thus, we obtain

$$
\operatorname{dim} \operatorname{Ker} L=\operatorname{dim} \operatorname{Im} Q=\operatorname{dim} \operatorname{Coker} L=1 .
$$

That is, $L$ is a Fredholm operator of index zero.

Now, we will prove that $K_{P}$ is the inverse of $\left.L\right|_{\operatorname{dom} L \cap K e r P}$. In fact, for $y \in \operatorname{Im} L$, we have

$$
K_{P} y(t)=-\frac{1}{\Gamma(\alpha)} \int_{0}^{t}(t-s)^{\alpha-1} y(s) d s+c_{0}
$$

where

$$
c_{0}=\frac{1}{\Gamma(\alpha+1)} \int_{0}^{1}(1-s)^{\alpha} y(s) d s
$$


It is easy to see that $L K_{P} y=y$. Moreover, for $x \in \operatorname{dom} L \cap \operatorname{Ker} P$, we get $x^{\prime}(0)=0$ and

$$
\begin{aligned}
K_{P} L x(t) & =I_{0^{+}}^{\alpha} D_{0^{+}}^{\alpha} x(t)-\left.I_{0^{+}}^{\alpha+1} D_{0^{+}}^{\alpha} x(t)\right|_{t=1} \\
& =x(t)-x(0)-\int_{0}^{1}(x(s)-x(0)) d s \\
& =x-P x .
\end{aligned}
$$

Combining (3.6) with (3.7), we know that $K_{P}=\left(\left.L\right|_{\operatorname{dom} L \cap \operatorname{Ker} P}\right)^{-1}$. The proof is complete.

Lemma 3.4 Assume $\Omega \subset X$ is an open bounded subset such that $\operatorname{dom} L \cap \bar{\Omega} \neq \emptyset$, then $N$ is $L$-compact on $\bar{\Omega}$.

Proof By the continuity of $f$, we see that $Q N(\bar{\Omega})$ and $K_{P}(I-Q) N(\bar{\Omega})$ are bounded. That is, there exist constants $A, B>0$ such that $|(I-Q) N x| \leq A$ and $\left|K_{P}(I-Q) N x\right| \leq B, \forall x \in \bar{\Omega}$, $t \in[0,1]$. Thus, one need only prove that $K_{P}(I-Q) N(\bar{\Omega}) \subset X$ is equicontinuous.

Let $K_{P, Q}=K_{P}(I-Q) N$, for $0 \leq t_{1}<t_{2} \leq 1, x \in \bar{\Omega}$, we get

$$
\begin{aligned}
& \left|\left(K_{P, Q} x\right)\left(t_{2}\right)-\left(K_{P, Q} x\right)\left(t_{1}\right)\right| \\
& \quad \leq \frac{1}{\Gamma(\alpha)}\left|\int_{0}^{t_{2}}\left(t_{2}-s\right)^{\alpha-1}(I-Q) N x(s) d s-\int_{0}^{t_{1}}\left(t_{1}-s\right)^{\alpha-1}(I-Q) N x(s) d s\right| \\
& \quad \leq \frac{A}{\Gamma(\alpha)}\left[\int_{0}^{t_{1}}\left(t_{2}-s\right)^{\alpha-1}-\left(t_{1}-s\right)^{\alpha-1} d s+\int_{t_{1}}^{t_{2}}\left(t_{2}-s\right)^{\alpha-1} d s\right] \\
& \quad=\frac{A}{\Gamma(\alpha+1)}\left(t_{2}^{\alpha}-t_{1}^{\alpha}\right) .
\end{aligned}
$$

Since $t^{\alpha}$ is uniformly continuous on $[0,1]$, we see that $K_{P, Q} N(\bar{\Omega}) \subset X$ is equicontinuous. Thus, we see that $K_{P, Q} N: \bar{\Omega} \rightarrow X$ is compact. The proof is completed.

Lemma 3.5 Suppose $\left(\mathrm{H}_{1}\right)$ and $\left(\mathrm{H}_{2}\right)$ hold, then the set

$$
\Omega_{0}=\{x \in \operatorname{dom} L: L x=\lambda N x, \lambda \in(0,1)\}
$$

is bounded.

Proof Take $x \in \Omega_{0}$, then $N x \in \operatorname{Im} L$. By (3.2), we have

$$
\sum_{i=1}^{m-2} \beta_{i} \int_{0}^{1} l_{i}(s) f(s, x(s)) d s=0
$$

Then, by the integral mean value theorem, there exists a constant $\xi \in(0,1)$ such that $f(\xi, x(\xi))=0$. So, from $\left(\mathrm{H}_{2}\right)$, we get $|x(\xi)| \leq B$. By Lemma 2.6 , one has

$$
\begin{aligned}
& x(t)=x(0)+\frac{1}{\Gamma(\alpha)} \int_{0}^{t}(t-s)^{\alpha-1} D_{0^{+}}^{\alpha} x(s) d s, \\
& x(\xi)=x(0)+\frac{1}{\Gamma(\alpha)} \int_{0}^{\xi}(\xi-s)^{\alpha-1} D_{0^{+}}^{\alpha} x(s) d s .
\end{aligned}
$$


Thus, we get

$$
\begin{aligned}
x(t)-x(\xi)= & \frac{1}{\Gamma(\alpha)} \int_{0}^{t}(t-s)^{\alpha-1} D_{0^{+}}^{\alpha} x(s) d s \\
& -\frac{1}{\Gamma(\alpha)} \int_{0}^{\xi}(\xi-s)^{\alpha-1} D_{0^{+}}^{\alpha} x(s) d s,
\end{aligned}
$$

which together with $\left(\mathrm{H}_{1}\right)$ implies that

$$
\begin{aligned}
|x(t)| & \leq|x(\xi)|+\frac{1}{\Gamma(\alpha)}\left\|D_{0^{+}}^{\alpha} x\right\|_{\infty} \cdot \frac{1}{\alpha}\left(t^{\beta}+\xi^{\beta}\right) \\
& \leq B+\frac{2}{\Gamma(\alpha+1)}\left\|D_{0^{+}}^{\alpha} x\right\|_{\infty} \\
& \leq B+\frac{2}{\Gamma(\alpha+1)} \cdot \max _{t \in[0,1]}|f(t, x(t))| \\
& \leq B+\frac{2}{\Gamma(\alpha+1)}\left(\|a\|_{\infty}+b_{1}\|x\|_{\infty}\right), \quad \forall t \in[0,1] .
\end{aligned}
$$

That is,

$$
\|x\|_{\infty} \leq B+\frac{2}{\Gamma(\alpha+1)}\left(\|a\|_{\infty}+b_{1}\|x\|_{\infty}\right)
$$

In view of $\frac{2}{\Gamma(\alpha+1)} b_{1}<1$, there exists a constant $D_{2}>0$ such that

$$
\|x\|_{\infty} \leq D_{2}
$$

Hence, $\Omega_{0}$ is bounded. The proof is complete.

Proof of Theorem 3.1 Set $C=\{x \in X: x(t) \geq 0, t \in[0,1]\}, \Omega_{1}=\{x \in X: r>|x(t)|>$ $\left.M\|x\|_{\infty}, t \in[0,1]\right\}$, and $\Omega_{2}=\left\{x \in X:\|x\|_{\infty}<R\right\}$, where $R=\max \left\{B, D_{2}\right\}$. Clearly, $\Omega_{1}, \Omega_{2}$ are open bounded subsets of $X$ and

$$
\bar{\Omega}_{1}=\left\{x \in X: r \geq|x(t)| \geq M\|x\|_{\infty}, t \in[0,1]\right\} \subset \Omega_{2} .
$$

From Lemma 3.3, Lemma 3.4, and Lemma 3.5, we see that the conditions (1) and (2) of Lemma 2.2 are satisfied.

Let $\gamma x(t)=|x(t)|$ for $x \in X$ and $J=I$. One can see that $\gamma$ is a retraction and maps subsets of $\bar{\Omega}_{2}$ into bounded subsets of $C$, which means that the condition (3) of Lemma 2.2 holds.

For $x \in \operatorname{Ker} L \cap \Omega_{2}$, we have $x(t) \equiv c$. Let

$$
H(c, \lambda)=c-\lambda|c|-\frac{\lambda \alpha}{\sum_{i=1}^{m-2} \beta_{i}\left(1-\eta_{i}^{\alpha}\right)} \sum_{i=1}^{m-2} \beta_{i} \int_{0}^{1} l_{i}(s) f(s,|c|) d s .
$$

From $H(c, \lambda)=0$, one has $c \geq 0$. Moreover, if $H(R, \lambda)=0$, we get

$$
0 \leq R(1-\lambda)=\frac{\lambda \alpha}{\sum_{i=1}^{m-2} \beta_{i}\left(1-\eta_{i}^{\alpha}\right)} \sum_{i=1}^{m-2} \beta_{i} \int_{0}^{1} l_{i}(s) f(s, R) d s,
$$


which contradicts $\left(\mathrm{H}_{2}\right)$. Thus $H(c, \lambda) \neq 0$ for $x \in \partial \Omega_{2}, \lambda \in[0,1]$. Hence

$$
\begin{aligned}
& \operatorname{deg}\left(\left.[I-(P+J Q N) \gamma]\right|_{\operatorname{Ker} L}, \operatorname{Ker} L \cap \Omega_{2}, 0\right) \\
& \quad=\operatorname{deg}\left(H(c, 1), \operatorname{Ker} L \cap \Omega_{2}, 0\right) \\
& \quad=\operatorname{deg}\left(H(c, 0), \operatorname{Ker} L \cap \Omega_{2}, 0\right) \\
& \quad=\operatorname{deg}\left(I, \operatorname{Ker} L \cap \Omega_{2}, 0\right) \\
& \quad=1 .
\end{aligned}
$$

So, the condition (4) of Lemma 2.2 holds.

Let $x \in \bar{\Omega}_{2} \backslash \Omega_{1}, t \in[0,1]$, we have

$$
\begin{aligned}
\Psi_{\gamma} x(t)= & \int_{0}^{1}|x(s)| d s+\frac{\alpha}{\sum_{i=1}^{m-2} \beta_{i}\left(1-\eta_{i}^{\alpha}\right)} \sum_{i=1}^{m-2} \beta_{i} \int_{0}^{1} l_{i}(s) f(s,|x(s)|) d s \\
& +\int_{0}^{1} k(t, s)\left[f(s,|x(s)|)-\frac{\alpha}{\sum_{i=1}^{m-2} \beta_{i}\left(1-\eta_{i}^{\alpha}\right)} \sum_{i=1}^{m-2} \beta_{i} \int_{0}^{1} l_{i}(\tau) f(\tau,|x(\tau)|) d \tau\right] d s \\
= & \int_{0}^{1}|x(s)| d s+\int_{0}^{1} G(t, s) f(s,|x(s)|) d s
\end{aligned}
$$

which together with $\left(\mathrm{H}_{3}\right)$ and (3.1) yields

$$
\Psi_{\gamma} x(t) \geq \int_{0}^{1}|x(s)| d s-\kappa \int_{0}^{1} G(t, s)|x(s)| d s=\int_{0}^{1}(1-\kappa G(t, s))|x(s)| d s \geq 0 .
$$

Thus, the condition (7) of Lemma 2.2 holds. In addition, we can prove the condition (6) of Lemma 2.2 holds too by a similar process.

Finally, we will show that the condition (5) of Lemma 2.2 is satisfied. Let $u_{0}(t) \equiv 1, t \in$ $[0,1]$, then $u_{0} \in C \backslash\{0\}, C\left(u_{0}\right)=\{x \in C: x(t)>0, t \in[0,1]\}$ and we can take $\sigma\left(u_{0}\right)=1$. For $x \in C\left(u_{0}\right) \cap \partial \Omega_{1}$, we have $x(t)>0, t \in[0,1], 0<\|x\|_{\infty} \leq r$, and $x(t) \geq M\|x\|_{\infty}, t \in[0,1]$. So, from $\left(\mathrm{H}_{4}\right)$, we obtain

$$
\begin{aligned}
\Psi x\left(t_{0}\right) & =\int_{0}^{1} x(s) d s+\int_{0}^{1} G\left(t_{0}, s\right) f(s, x(s)) d s \\
& \geq M\|x\|+\int_{0}^{1} G\left(t_{0}, s\right) h(x(s)) d s \\
& =M\|x\|+\int_{0}^{1} G\left(t_{0}, s\right) \frac{h(x(s))}{x(s)} x(s) d s \\
& \geq M\|x\|+\frac{h(r)}{r} \int_{0}^{1} G\left(t_{0}, s\right) x(s) d s \\
& \geq M\|x\|+\frac{h(r)}{r} \int_{0}^{1} G\left(t_{0}, s\right) M\|x\| d s \\
& \geq M\|x\|+(1-M)\|x\| \\
& =\|x\| .
\end{aligned}
$$

Then the condition (5) of Lemma 2.2 holds. 
Consequently, by Lemma 2.2, the equation $L x=N x$ has at least one solution $x^{*} \in$ $C \cap\left(\bar{\Omega}_{2} \backslash \Omega_{1}\right)$. Namely, FBVP (1.1) has at least one positive solution in $X$. The proof is complete.

\section{Example}

We consider the following FBVP:

$$
\left\{\begin{array}{l}
-D_{0^{+}}^{\frac{3}{2}} x(t)=\sin t-\frac{1}{10} x(t)+10+\sin x(t), \quad t \in[0,1] \\
x^{\prime}(0)=0, \quad x(1)=x\left(\frac{1}{2}\right) .
\end{array}\right.
$$

Thus, we have

$$
\begin{aligned}
& l(s)= \begin{cases}\sqrt{1-s}-\sqrt{\frac{1}{2}-s}, & 0 \leq s \leq \frac{1}{2}, \\
\sqrt{1-s}, & \frac{1}{2} \leq s \leq 1,\end{cases} \\
& G(t, s)= \begin{cases}\frac{1}{\Gamma\left(\frac{5}{2}\right)}(1-s)^{\frac{3}{2}}+\frac{3\left(\Gamma\left(\frac{7}{2}\right)-1+\left(\frac{5}{2}\right) t^{\frac{3}{2}}\right)}{2 \Gamma\left(\frac{7}{2}\right)\left(1-\left(\frac{1}{2}\right)^{\frac{3}{2}}\right)} l(s), & 0 \leq t \leq s \leq 1, \\
\frac{1}{\Gamma\left(\frac{5}{2}\right)}(1-s)^{\frac{3}{2}}-\frac{1}{\Gamma\left(\frac{3}{2}\right)}(t-s)^{\frac{1}{2}}+\frac{3\left(\Gamma\left(\frac{7}{2}\right)-1+\left(\frac{5}{2}\right) t^{\frac{3}{2}}\right)}{2 \Gamma\left(\frac{7}{2}\right)\left(1-\left(\frac{1}{2}\right)^{\frac{3}{2}}\right)} l(s), & 0 \leq s \leq t \leq 1 .\end{cases}
\end{aligned}
$$

Moreover, $f(t, u) \geq 8-\frac{1}{10} u \geq-\frac{1}{4} u$ for all $u \geq 0$, and $l(s) \leq 1, G(t, s) \leq 4, \kappa=-\frac{1}{4}$. So, we can find that $\left(\mathrm{H}_{1}\right),\left(\mathrm{H}_{2}\right)$, $\left(\mathrm{H}_{3}\right)$ hold. Next, we take $t_{0}=0, h(x)=x$, and $M=\frac{2}{3}$, thus $G(0, s)=\frac{1}{\Gamma\left(\frac{5}{2}\right)}(1-s)^{\frac{3}{2}}+\frac{3\left(\Gamma\left(\frac{7}{2}\right)-1\right)}{2 \Gamma\left(\frac{7}{2}\right)\left(1-\left(\frac{1}{2}\right)^{\frac{3}{2}}\right)} l(s), 0 \leq s \leq 1$, and $\int_{0}^{1} G(0, s) d s=1$. Then $\left(\mathrm{H}_{4}\right)$ is satisfied. According to the above points, by Theorem 3.1, we can conclude that FBVP (4.1) has at least one positive solution.

\section{Competing interests}

The authors declare that they have no competing interests.

\section{Authors' contributions}

All authors contributed equally to the writing of this paper. All authors read and approved the final manuscript.

\section{Acknowledgements}

The authors would like to thank the referees very much for their helpful comments and suggestions. This work was supported by the National Natural Science Foundation of China (11271364).

Received: 25 March 2015 Accepted: 29 June 2015 Published online: 06 August 2015

\section{References}

1. Glockle, WG, Nonnenmacher, TF: A fractional calculus approach of self-similar protein dynamics. Biophys. J. 68, 46-53 (1995)

2. Oldham, KB, Spanier, J: The Fractional Calculus. Academic Press, New York (1974)

3. Diethelm, K, Freed, AD: On the solution of nonlinear fractional order differential equations used in the modeling of viscoplasticity. In: Keil, F, Mackens, W, Voss, H, Werther, J (eds.) Scientific Computing in Chemical Engineering II. Computational Fluid Dynamics, Reaction Engineering and Molecular Properties, pp. 217-224. Springer, Heidelberg (1999)

4. Agarwal, RP, O'Regan, D, Stanek, S: Positive solutions for Dirichlet problems of singular nonlinear fractional differential equations. J. Math. Anal. Appl. 371, 57-68 (2010)

5. Bai, Z, Lü, H: Positive solutions for boundary value problem of nonlinear fractional differential equation. J. Math. Anal. Appl. 311, 495-505 (2005)

6. Kaufmann, ER, Mboumi, E: Positive solutions of a boundary value problem for a nonlinear fractional differential equation. Electron. J. Qual. Theory Differ. Equ. 2008, 3 (2008)

7. Zhang, S: Positive solutions for boundary-value problems of nonlinear fractional differential equations. Electron. J. Differ. Equ. 2006, 36 (2006)

8. Jafari, H, Gejji, VD: Positive solutions of nonlinear fractional boundary value problems using Adomian decomposition method. Appl. Math. Comput. 180, 700-706 (2006) 
9. Liang, S, Zhang, J: Positive solutions for boundary value problems of nonlinear fractional differential equation. Nonlinear Anal. 71, 5545-5550 (2009)

10. Bai, Z, Zhang, Y: Solvability of fractional three-point boundary value problems with nonlinear growth. Appl. Math. Comput. 218, 1719-1725 (2011)

11. Kosmatov, N: A boundary value problem of fractional order at resonance. Electron. J. Differ. Equ. 2010, 135 (2010)

12. Jiang, $W$ : The existence of solutions to boundary value problems of fractional differential equations at resonance. Nonlinear Anal. 74, 1987-1994 (2011)

13. Yang, A, Wang, $\mathrm{H}$ : Positive solutions of two-point boundary value problems of nonlinear fractional differential equation at resonance. Electron. J. Qual. Theory Differ. Equ. 2011, 71 (2011)

14. Chen, Y, Tang, X: Positive solutions of fractional differential equations at resonance on the half-line. Bound. Value Probl. 2012, 64 (2012)

15. O'Regan, D, Zima, M: Leggett-Williams norm-type theorems for coincidences. Arch. Math. 87, 222-244 (2006)

16. Mainardi, F: Fractional calculus: some basic problems in continuum and statistical mechanics. In: Carpinteri, A Mainardi, F (eds.) Fractals and Fractional Calculus in Continuum Mechanics, pp. 291-348. Springer, Wien (1997)

17. Metzler, F, Schick, W, Kilian, HG, Nonnenmacher, TF: Relaxation in filled polymers: a fractional calculus approach. J. Chem. Phys. 103, 7180-7186 (1995)

18. Lakshmikantham, V, Leela, S, Vasundhara Devi, J: Theory of Fractional Dynamic Systems. Cambridge Academic Publishers, Cambridge (2009)

\section{Submit your manuscript to a SpringerOpen ${ }^{\circ}$ journal and benefit from:}

- Convenient online submission

- Rigorous peer review

- Immediate publication on acceptance

- Open access: articles freely available online

- High visibility within the field

- Retaining the copyright to your article 